\title{
Identidad docente y estrategias de resolución de incidentes críticos en contextos universitarios de alta diversidad sociocultural
}

\author{
CRISTA WeISE Y SÒNIA SÀnCHEZ-BuSQuÉS* \\ Universitat Autònoma de Barcelona
}

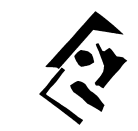

Resumen

Este estudio identifica las estrategias de los profesores cuando se enfrentan a incidentes críticos (IC) en contextos de alta diversidad sociocultural, reconociendo el peso de la identidad y de las emociones en la toma de decisiones, así como la efectividad de las mismas para promover cambios en las prácticas docentes. En el estudio de naturaleza cualitativa, fueron entrevistados 23 profesores universitarios. Los resultados indican que las emociones asociadas a un IC, generan una situación de desequilibrio que puede obstaculizar la actividad cuando son de naturaleza negativa. Al mismo tiempo, en estas situaciones es posible la revisión y reconstrucción de la propia identidad docente. En cuanto a las estrategias empleadas por los docentes ante los IC, éstas fueron mayoritariamente inmediatas e incidieron en aspectos muy puntuales, lo que derivó en actuaciones reactivas y poco eficaces. Pese a ello, la mayoría de los profesores no modificaron sus posiciones iniciales y fueron excepcionales las situaciones en las que el despliegue de estrategias y la reflexión posterior a un determinado IC permitió cambios estructurales que impactaron en alguna dimensión de la identidad del profesor como sus emociones y prácticas.

Palabras clave: Incidentes críticos, identidad docente, contextos socioculturales, educación superior, emociones en el docente, formación del profesorado.

\section{Teacher identity and resolution strategies of critical incidents in university context with high cultural diversity}

\begin{abstract}
This study identifies the strategies that teachers employ when faced with critical incidents (CI) in highly diverse sociocultural contexts, recognizing the weight of identity and emotions in decision-making and their effectiveness to promote changes in teaching practice. It is a qualitative study with 23 university teachers. The results indicate that the emotions produced by a CI, create a situation of imbalance, accompanied by negative emotions that binder teachers' activity. The study states that there is a need to review the situation and to open the possibility of teachers' identity reconstruction. With respect to the strategies employed by teachers when faced with CI, these were mostly immediate and affected very specific aspects, resulting in reactive performance and a choice of ineffective strategies. Despite the poor effectiveness of their strategies, in most cases, teachers did not change their initial positions. IC deployment strategies and subsequent reflection that allow an opening to structural changes that impact on teacher identity (emotional and practical) are an exception.

Keywords: Critical incidents, teacher identity, sociocultural context, higher education, teacher's emotions, teacher training.
\end{abstract}

\footnotetext{
*Universitat Autònoma de Barcelona/SINTE Seminari Interuniversitari d'Investigació i Estratègies d'Aprenentatge. http://www.sinte.es

Correspondencia con las autoras: Crista Weise. Universitat Autònoma de Barcelona, Departamento de Psicología Básica, Evolutiva y de la Educación, Edificio G6. Despacho 263. Facultad de Educación, Campus de la UAB. 08193 Cerdanyola del Vallès (Barcelona). Telf. 3493581 3187. E-mail: crista.weise@uab.cat

Original recibido: 25 de mayo de 2013. Aceptado: 3 de octubre de 2013.
} 
562 Cultura y Educación, 2013, 25 (4), pp. 561-576

\section{INTRODUCCIÓN}

Uno de los aspectos más debatidos en el campo educativo, aunque no por ello resuelto, es el relativo a la posibilidad de promover cambios en las prácticas docentes cuando dichas prácticas se revelan poco eficaces o ajustadas a las demandas del contexto.

El análisis de las propuestas y modelos de formación continua del profesorado muestra posiciones dicotómicas y, a menudo poco articuladas, que pueden esquematizarse en función de su carácter preferentemente conceptual o experiencial (Sutherland, Howard, y Markauskaite, 2010). En el primer caso, los problemas aparecen cuando las formas de interpretar y actuar sobre la realidad educativa se adquieren de manera descontextualizada. En el segundo caso, cuando no se garantiza una integración efectiva entre teoría y práctica y su posterior aplicación. Además algunos autores (Cope y Stephen, 2001; Ebbut, Robson y Worrall, 2000; Mewborn y Stanulis, 2000) señalan que en la mayoría de los contextos formativos dominantes, se elaboran teorías, concepciones y creencias de carácter implícito que con frecuencia permanecen a un nivel poco consciente por lo que resulta difícil que puedan ser redescritas en aras de una toma de decisiones más estratégica. Lamentablemente, ambas posiciones no llegan habitualmente a integrarse de una manera eficaz (Rice, 2002). La investigación sobre el uso de incidentes críticos (a partir de ahora IC) en situaciones de formación resulta una alternativa interesante a esta falta de integración.

Los IC se definen como sucesos que desestabilizan la acción del profesor al superar un determinado umbral emocional como consecuencia de una disonancia entre una situación conflictiva que emerge de forma inesperada y las expectativas e intenciones de este profesor. Los IC remiten siempre a situaciones concretas y específicas, en las que los conflictos se interpretan de forma contextualizada y cuyo análisis pone de manifiesto aspectos de la identidad del profesor vehiculizados a través de sus decisiones, estrategias y emociones.

Si queremos modificar las prácticas docentes a partir de un proceso formativo, requerimos que el profesor pueda tomar consciencia de sus acciones educativas, no sólo para distanciarse y reflexionar sobre sus actuaciones, sino para redescribirlas a la luz de los conocimientos y las experiencias que le propone el formador.

Para que un cambio en la práctica docente sea duradero es necesario que el IC modifique la identidad del profesor ya que la identidad de éste funciona como el marco general de interpretación y de actuación. Cambiar la identidad implicará cambiar los implícitos desde los cuales el profesor actúa, posibilitando una interpretación del problema desde otros marcos y en consecuencia, intentar soluciones que conduzcan a cambios duraderos. Modificar lo que se hace en clase puede promover cambios en la identidad del docente (Beth y Sharma, 2008; Day, Kington, Stobart y Sammons, 2006; Enyedy, Goldberg y Welsh, 2006; Monereo, Badía, Bilbao, Cerrato y Weise, 2009; Roberts, 2007). Bajo estas premisas, el estudio que presentamos tiene por objetivo profundizar en las relaciones entre las emociones y las estrategias con que enfrentan los profesores los incidentes críticos y sus posibles relaciones con cambios en la identidad docente.

\section{La identidad del profesor y la práctica docente}

La identidad profesional resulta un constructo esencial para comprender las instituciones educativas y lo que en ellas ocurre (Gee, 2001), y la investigación ha puesto de manifiesto su relación con las emociones (Van Veen y Lasky, 2005), con la capacidad de agencia del profesor (Lasky, 2005; Nixon, 1996), con la efectividad de la enseñanza (Hopper, 2008; Settlage, Southerland, Smith y Ceglie, 2009), y con el grado de compromiso de los profesores en las actividades educativas (Day, Elliot, y Kington, 2005).

Si bien existen enfoques diversos respecto al tratamiento de la identidad, en este estudio adoptamos una posición integradora de carácter socioconstructivista que define la identidad del profesor como un conjunto de representaciones que éste tiene 
sobre sí mismo, frente a sí mismo y a los demás, cambiante según los contextos de práctica (identidad en acción) pero con componentes relativamente estables en el tiempo (identidad narrativa) en relación con su rol profesional, las emociones vinculadas a su práctica y las percepciones sobre su contexto institucional, político, social y cultural. Dichas representaciones, así como los procesos de reconstrucción y redescripción de las mismas, dan sentido a su práctica y justifican sus decisiones y acciones en el día a día (Veiravé, Ojeda, Nuñez y Delgado, 2006). Así pues, además de estar constituida por representaciones y ser contextual, la identidad profesional también posee una dimensión emocional, que tiene que ver con deseos, placeres, dolores y pérdidas en relación al ejercicio de la docencia (Day et al., 2005); esta dimensión está asociada al compromiso, motivación e implicación del profesor con las prácticas que desarrolla en el aula (Heargreaves, 1998).

En este trabajo centramos la mirada en la actuación docente, por lo tanto, en la identidad en acción, en contextos universitarios socioculturalmente diversos. Las variadas demandas y complejas exigencias de estos contextos, a menudo cuestionan y desafían la identidad y los recursos del profesorado y le obligan a regular sus concepciones, estrategias y sobre todo sus emociones para responder a las mismas de forma ajustada.

\section{Las emociones en la práctica docente}

Si bien la relación entre las emociones, la identidad y el cambio educacional ha sido escasamente estudiada (Lasky, 2005; Sutton y Wheatley, 2003), varios investigadores han dado cuenta de la importancia de dicha relación y su repercusión con la práctica docente (Reio, 2005), poniendo de manifiesto que tienen un rol central en el proceso cotidiano de toma de decisiones del profesor (Ria, Sève, Saury, Theureu y Durand, 2003), en su desarrollo profesional y en el proceso de formación de la identidad (Shapiro, 2010).

Las sensaciones, las emociones y los sentimientos están asociados a modos de percibir determinadas situaciones. Las sensaciones se corresponden con lo que Ria y colaboradores (Ria et al., 2003) han denominado un modo potencial de existencia, es decir, situaciones que posibilitan una apertura del sujeto pero que en el momento en que se producen carecen de significado específico. La sensación posibilita la evocación de experiencias pasadas y su potencial transformación. Las emociones básicas ${ }^{1}$, se manifiestan de forma delimitada en el tiempo, pueden ser expresadas y definidas por el sujeto, son de carácter transitorio y generalmente surgen como respuesta a situaciones o problemas concretos que están sucediendo en ese momento y por ello pueden asociarse a un modo de existencia actual (Ria et al., 2003). Finalmente, los sentimientos son más estables en el tiempo ya que implican una reflexión, un proceso de redescripción mediante el cual el sujeto encuentra unas ciertas regularidades o generalizaciones y que Ria y colaboradores han denominado modo virtual de existencia por su menor nivel de sujeción a una situación, tiempo y lugar.

Esta distinción resulta interesante en cuanto permite distinguir el tipo de emociones vinculadas a la identidad del docente y especialmente aquellas relacionadas con situaciones específicas como son los IC. En este artículo, nos interesan las emociones asociadas a los diferentes IC y a las estrategias que despliega el profesor para resolverlos. En este sentido, usamos el término emociones básicas para aquellas que son propias de la identidad en acción que aparecen en los IC (modo actual de existencia) y usamos el término sentimientos para aquellas emociones redescritas por el profesor que aparecen asociadas a la identidad narrativa (modo virtual de existencia).

Algunos estudios sostienen que las emociones negativas distraen de las metas educativas, obstaculizan los procesos de cambio, incrementan las resistencias, afectan a su capacidad de resolución de problemas y disminuyen la toma de riesgos por parte del profesor; 
564 Cultura y Educación, 2013, 25 (4), pp. 561-576

mientras que las positivas las favorecen e incrementan la efectividad del docente y su capacidad de agencia, dando como resultado una enseñanza más eficiente (Kelchtermans, 2005; Lasky, 2005; Reio, 2005; Sutton y Wheatley, 2003). Por otra parte, identidad, emociones y procesos de cambio están directamente relacionados (Heargreaves, 1998; Scott y Sutton, 2009), lo que reafirma el valor de los IC como estrategia de formación, ya que al tener un impacto emocional generan una situación de desequilibrio y un cuestionamiento de la situación, lo que, a su vez, abre la posibilidad de la deconstrucción y reconstrucción de la identidad (Scott y Sutton, 2009; Sutton y Wheatley, 2003).

\section{Las estrategias docentes frente al IC}

Recordemos que hemos definido un IC como una situación que interpela al profesor y que le obliga a introducir en su práctica docente formas de actuación imprevistas. Por lo tanto, son situaciones que tienen un componente emocional importante y que pueden afectar a su identidad profesional (Monereo et al., 2009).

Sin embargo el desajuste que provoca un IC depende de las características del profesor por lo que una situación puede representar un IC para un profesor pero podría no serlo para otro. Lo crítico viene definido por la manera en que el profesor se representa el evento (Tripp, 1994). Consecuentemente, los IC aparecen ante eventos concretos y situados en el tiempo y el espacio que manifiestan situaciones desestabilizantes muy acotadas, pero que, en muchas ocasiones, son indicativos de conflictos subyacentes más profundos (Burgum y Bridge, 1997). Cuando el IC dispara emociones en el profesor, éste las asocia a situaciones pasadas, a cómo se percibe y el rol que desempeña, y de esta forma impacta en dimensiones de su identidad, generando como hemos mencionado una situación desestabilizadora pero también abriendo oportunidades para el cambio.

FIGURA 1

Esquema de análisis de IC

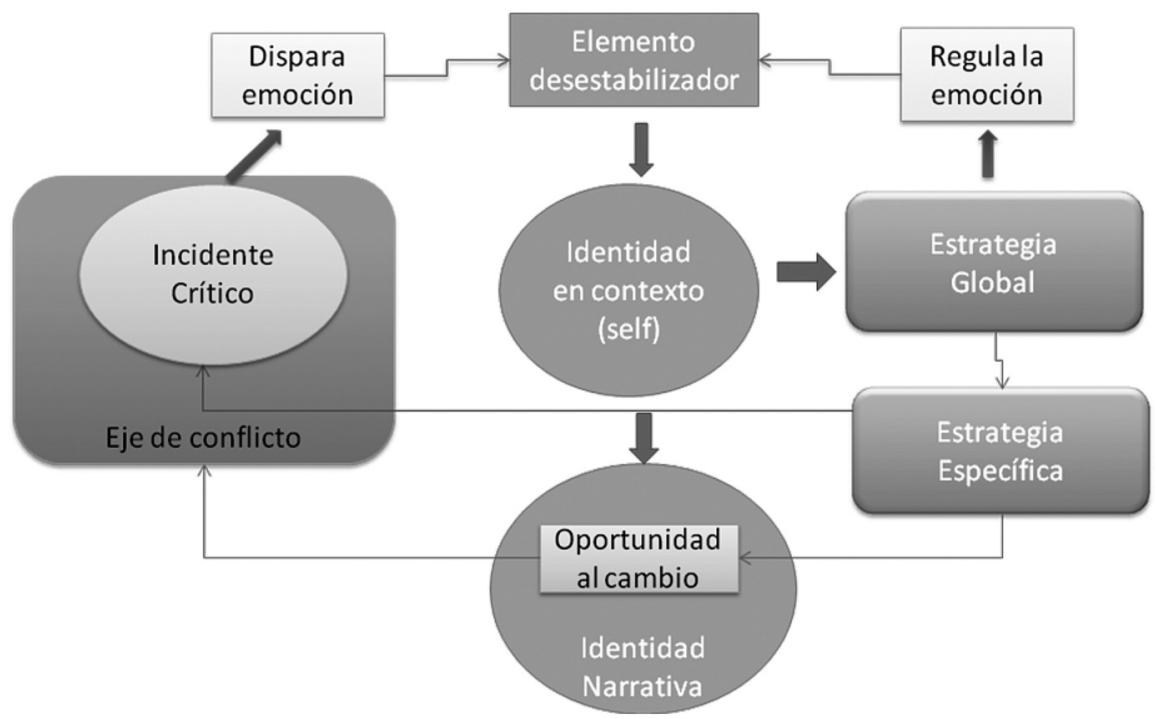

Como puede observarse en la figura 1, cuando el profesor se encuentra frente a un IC lo interpreta para reaccionar al impacto emocional en un lapso muy corto de tiempo, tomando decisiones y desplegando estrategias para resolverlo. Estas estrategias se ejecutan a dos niveles: global y específico. Así, en primer lugar se desarrolla una línea de acción general -estrategia global (en adelante EG)-, que permite al profesor enfrentar la situación, regular 
las emociones de manera manejable y establecer una línea de actuación frente al problema. Dentro de esta EG, el profesor toma decisiones y acciones concretas de segundo nivel, más específicas, que considera coherentes (aunque para un observador externo no necesariamente lo sean) con la EG y que buscan reconducir la situación y reorientarla hacia el logro de sus propósitos; a estas estrategias las llamamos estrategias específicas (en adelante EE). La EG da sentido a la EE y establece su funcionalidad.

En base a las consideraciones precedentes, el estudio que presentamos tiene los siguientes objetivos: a) identificar situaciones de afrontamiento y estrategias tipo empleadas por los profesores para resolver IC referidos al carácter socioculturalmente diverso de sus estudiantes, b) identificar qué emociones aparecen en los profesores frente a los diferentes IC, qué decisiones toma y cuál es el sentido que tienen en el IC y c) analizar si esas decisiones permiten abrir posibilidades de cambio más profundos, es decir, si hay un impacto en la identidad del profesor universitario.

\section{MÉTODO}

El estudio adopta un diseño descriptivo e interpretativo en el que se analizan los relatos de los profesores sobre cómo interpretan los incidentes críticos a los que se enfrentan.

\section{Participantes}

Mediante un muestreo intencional, se eligieron 23 profesores a partir de los siguientes criterios de selección: la diversidad en relación a la afiliación institucional y el área de conocimiento, y la experiencia en la docencia universitaria (más de 5 años). Suponíamos que el hecho de que contaran con experiencia evitaría que fuesen excesivamente vulnerables a incidentes propios de profesores noveles y reconocieran mejor aquellos asociados a la diversidad sociocultural.

Finalmente, la muestra se compuso de 20 profesores del área de ciencias sociales, humanidades y educación y 3 del área tecnológica; 17 eran de una universidad pública y 6 de una privada. Todos ellos participaron en la investigación de forma voluntaria y firmaron su consentimiento después de haber sido debidamente informados de los objetivos de la misma.

\section{Procedimiento}

En una primera etapa, los profesores construyeron un relato en el que explicaban quiénes eran, con qué problemas asociados a la diversidad sociocultural se habían tenido que enfrentar y cómo los habían resuelto. A partir de los datos extraídos de las narraciones de los profesores, se realizó una entrevista en profundidad de carácter semi-estructurado en la que los entrevistados manifestaron sus preocupaciones y explicaron diferentes IC asociados a la diversidad sociocultural a los que se habían enfrentado en los últimos 2 años de su actividad.

Todas las entrevistas fueron grabadas y posteriormente transcritas íntegramente para su posterior codificación y análisis con el programa informático de análisis cualitativo de datos Atlas Ti. Para el análisis se siguió el procedimiento propuesto por Miles y Huberman (1984).

Se realizó un primer procesamiento y codificación en Atlas Ti y, en una segunda etapa, se procedió a una segunda recolección de datos, (nueva ronda de entrevistas semiestructuradas) para complementar la información y redescribir los IC.

El proceso de codificación y validación de las categorías se llevó a cabo con un grupo de 25 profesores de características similares a la muestra, en una reunión de expertos que duró tres jornadas de cuatro horas cada una. Los expertos recibieron las citas completas seleccionadas con la respectiva referencia de su codificación, la categoría a la que pertenecían y la definición operacional empleada en ese código. Su valoración se realizó a partir de dos criterios: a) si la categoría y su definición operacional era pertinente y b) si las citas seleccio- 
566 Cultura y Educación, 2013, 25 (4), pp. 561-576

nadas correspondían a esa categoría y por tanto si había acuerdo o desacuerdo con esa codificación. Los acuerdos se obtuvieron a través de la generación de consensos y en los casos en los que el grupo señaló dudas se eliminaron o modificaron las categorías. El mismo proceso se repitió con 120 estudiantes de último año en una jornada. Aunque las percepciones de los estudiantes no eran parte de nuestro objeto de estudio, quisimos contrastar si los incidentes y la manera en que eran definidos e interpretados por los profesores, estaban en concordancia con sus percepciones.

Todo el proceso de recogida de datos se realizó en un intervalo de tiempo de seis meses. Las entrevistas fueron llevadas a cabo por una investigadora, autora de este artículo y por un profesor colaborador perteneciente al centro público y conocedor de los objetivos y los supuestos teóricos del estudio.

\section{Análisis de datos}

Siguiendo las recomendaciones de Pandit, Corbin y Strauss (Corbin y Strauss, 1990; Pandit, 1996) bajo el paradigma de la grounded theory, se procesó la información mediante aproximaciones sucesivas a las categorías finales con la ayuda del programa Atlas.Ti 5.0, como ya hemos mencionado. Se consideraron 352 citas referidas a la actuación del profesor. Se codificaron un total de 43 IC y 96 citas referidas a estrategias específicas.

Para su análisis, se partió de categorías generales pre-definidas a partir del marco teórico inicial y se optó por una estrategia combinada de codificación: Codificación top-down (categorías predefinidas) y codificación down-top (categorías emergentes).

El procedimiento seguido para el establecimiento de categorías emergentes consistió en: a) clasificar las estrategias empleadas por los profesores, distinguiendo las Estrategias Globales (EG) y las Estrategias Específicas (EE); b) clasificar las situaciones tipo de resolución contrastando con estudios previos; c) identificar las emociones que mencionaron los profesores durante los IC; d) analizar la resolución del IC a partir del esquema teórico propuesto (Figura 1).

Para la presentación de la información elaboramos tablas descriptivas de los componentes del IC tal y como se recoge en la tabla I.

TABLA I

Ejemplo de cuadro de análisis

\begin{tabular}{|c|c|c|c|c|}
\hline IC & Emoción & Actuación docente & EG & $\mathrm{EE}$ \\
\hline $\begin{array}{l}\text { Los estudiantes } \\
\text { no } \\
\text { comprendieron } \\
\text { un texto }\end{array}$ & Frustración & $\begin{array}{l}\text { Aunque cree } \\
\text { que el texto era } \\
\text { fácil lo } \\
\text { sustituyó por } \\
\text { actividad } \\
\text { práctica }\end{array}$ & $\begin{array}{l}\text { Estrategia } \\
\text { localizada }\end{array}$ & $\begin{array}{l}\text { Actividad } \\
\text { práctica }\end{array}$ \\
\hline
\end{tabular}

El análisis se hizo recogiendo la información sobre la totalidad de IC, ordenados de acuerdo a 6 ejes de conflictos socioculturales (lógicas de pensamiento, lenguas, expresión y comunicación, concepciones del tiempo, contextualización, discriminación, participación) identificados por Weise en un estudio previo con la misma población (Weise, 2011).

\section{RESULTADOS}

Los resultados se presentan en dos apartados; el primero hace referencia a las situaciones y estrategias tipo empleadas por los docentes mientras que en el segundo se desglosa el análisis de los IC en relación a las emociones que conllevan y al tipo de decisiones que toman los profesores para su resolución, estableciendo relaciones entre emociones, estrategias y posibilidad de cambio. 


\section{Situaciones de resolución y estrategias tipo}

A partir del esquema de análisis propuesto (ver Figura 1), al examinar la actuación de los profesores de la muestra frente a los 43 IC reportados encontramos las siguientes situaciones y estrategias de resolución:

\section{Situación 1: EG (A) no se despliega estrategia (No hay cambio)}

En estas situaciones, el docente identifica el IC, pero no se siente responsable o suficientemente interpelado y por lo tanto ignora el problema y no se despliega una EG. No llega a haber desestabilización suficiente y por consiguiente no se favorece cambio alguno.

Un ejemplo de este tipo de situación es el siguiente:

Intenté en algún momento hacer un seguimiento serio para mejorar la velocidad de lectura pero ahí noté que hacía falta alguna otra palanca a la que yo no tenía acceso y tampoco quería inmiscuirme mucho en eso, entonces al ver que había una cierta resistencia, yo lo dejé, entonces yo dije yo se las presento [las lecturas], ellos voluntariamente deciden hacerlo; pero si quieren hacerlo es cosa suya, ya no es mi responsabilidad como docente hacer ese seguimiento. No me voy a hacer un problema por ello y por eso lo dejé allí. $17: 17[36]^{2}$

\section{Situación 2: $E G(B)$ de protección o evitación (Decisión de no acción-no bay cambio)}

En esta situación, la estrategia de resolución adoptada se dirige principalmente a recuperar la estabilidad emocional o el control de la situación, independientemente de si esto está en sintonía con los objetivos del profesor. La EG es de autoprotección, y por lo tanto se evita el IC por vía de la evasión del problema. El incidente es crítico, interpela al docente, pero intencionalmente se evita la acción. No hay cambio.

Un ejemplo de este tipo de situación es el siguiente:

Al grupo de esta muchacha le tocó exponer Bourdieu y no podía ni atrás ni adelante, era evidente que ella se aprendió de memoria su parte y listo; y yo hice como que ya expuso su parte, dejé pasar y acepté la exposición, no hice nada más y obviamente no entendió nada. Me sentí sin saber qué hacer y decía yo o les quito Bourdieu de la materia o qué hago, realmente no sé, y ese es mi problema no se si tratarlas de manera especial a ellas o exigirles de manera similar a los estudiantes que hay [refiriéndose a los otros compañeros de aula] y por no equivocarme dejé pasar. 1:36[236]

\section{Situación 3: EG (C) localizada (Estrategia localizada o inadecuada)}

En estas situaciones, el docente se siente interpelado, se responsabiliza y toma decisiones que considera adecuadas para la situación. El docente detecta el IC y piensa que lo ha resuelto. No obstante, si bien su respuesta podría solventar temporalmente el conflicto, a medio y largo plazo la posibilidad de que la situación se reproduzca es elevada. En este caso, el profesor enfrenta el problema, aunque pone en juego una EG restringida o inadecuada que favorece la resolución inmediata o sintomática del IC pero que no necesariamente conduce a una solución sostenible. El cambio (cuando se produce) no modifica los patrones de acción del profesor.

Un ejemplo de este tipo de situación es el siguiente:

\footnotetext{
Era una persona que le costaba y yo he tomado en cuenta que cada ser humano es distinto, a un citadino puedes exigirle más, pero a un quechua, en realidad para que llegue a comprender muchas cosas ha dado grandes saltos, [...] entonces yo he tomado en cuenta, o sea el problema ha sido que no ha podido comprender, pero frente a sus limitaciones yo he visto ese intento de comprender. Y lo que hecho es simplificar, pedirle tareas sencillas con menos inversión cognitiva por decir algo y que así pueda promediar. $2: 31[170]$
}

\section{Situación 4: $E G(D)$ potencialmente ajustada (Impacto en la identidad, respuesta ajustada)}

En esta situación, el profesor se siente interpelado, pero además el IC consigue que el docente recapacite, se auto-atribuya la responsabilidad y abre la puerta a una re-concep- 
tualización de sus concepciones, prácticas y sentimientos asociados (Monereo, 2010) aumentando sus posibilidades de dar una respuesta más ajustada al contexto.

Un ejemplo de este tipo de situación es el siguiente:

Los alumnos que tienen como lengua materna un idioma nativo, si bien hablan sin dificultad el castellano y se han acostumbrado a escuchar y manejar la teoría sociológica en este idioma, no pueden aplicar esa herramienta cuando van a hacer prácticas en lugares de habla quechua; entonces me ha resultado muy útil, hacer la pregunta "cómo me preguntarías eso en quechua", "cómo me explicarías eso en quechua", no es necesario que el docente domine el idioma, escuchando la explicación del alumno uno puede identificar palabras que permiten explicar o comprender lo que está en discusión. Mi forma de actuar ha sido utilizar el recurso del lenguaje (idioma nativo) para ayudar a los estudiantes a traducir la teoría sociológica y los conceptos a un lenguaje cotidiano y a su propia lógica. $18: 10[23]$

Aunque las posibilidades de combinación son variadas, estas cuatro situaciones son representativas del tipo de escenarios y formas de resolución que hemos encontrado en los datos analizados. Así, del total de IC, 3 se caracterizaron como EG (A), 11 como EG (B), 25 EG (C) y 5 como EG (D).

En cuanto a los resultados relativos a las categorías emergentes, se identificaron 10 tipos de EE que suelen utilizar los profesores: (1) reflexión/mediación, (2) tiempo extra, (3) actividad práctica, (4) diversificación métodos, (5) incentivación, (6) organización del grupo, (7) recurso de la cultura, (8) simplificación/facilitación, (9) no actuación, (10) no dispone de estrategia.

Las EE más utilizadas por el conjunto de profesores analizados pueden consultarse en la tabla II.

TABLA II

Frecuencia de uso de Estrategias Específicas docentes

\begin{tabular}{ll}
\hline $\begin{array}{c}\text { Estrategia del profesor } \\
\text { frente al IC }\end{array}$ \\
\hline 1 & Reflexión/mediación \\
2 & Tiempo extra \\
3 & Actividad práctica \\
4 & Diversifica métodos
\end{tabular}

5 Incentiva

6 Organiza el grupo

$7 \quad$ Recurso cultura

8 Simplifica/facilita

9 No actúa

10 No dispone

\section{Definición}

$N$

El profesor utiliza la reflexión, o actúa como mediador.

El profesor dedica un tiempo adicional.

El profesor propone una actividad de aplicación.

El profesor alterna o prueba diferentes métodos o varía las estrategias probando cuáles le funcionan o complementa su uso.

El profesor realiza intervenciones directas para estimular a los estudiantes a participar.

El profesor modifica puntualmente la forma de organización del grupo.

El profesor toma algún ejemplo, dato, contenido o estrategia de las culturas de sus estudiantes.

El profesor opta por rebajar la exigencia o simplificar tareas y explicaciones.

El profesor continua haciendo lo que tenía previsto.

El profesor no tiene estrategias para poner en juego, o no sabe cómo actuar.

\section{7}

2

2

3

4

\section{Análisis de la actuación docente, emociones y estrategias de resolución}

En las tablas III, IV, V, VI, VII y VIII se recogen los resultados relativos a la actuación docente frente al IC. En ellas se describe cuál fue el evento desestabilizador, qué hizo el 
Identidad docente e incidentes críticos en contextos de alta diversidad / C. Weise y S. Sànchez-Busqués

docente frente a este evento, qué emociones relató, cuáles fueron las EG que puso en juego y cuál fue la EE que utilizó para afrontar el IC.

Eje de lógicas de pensamiento

TABLA III

Eje lógicas de pensamiento

\begin{tabular}{ll}
\hline $\begin{array}{l}\text { Definición del eje de } \\
\text { conflicto }\end{array}$ & $\begin{array}{l}\text { Trata de un choque de sistemas culturales en el que entran en } \\
\text { contradicción modos diferentes de pensamiento asociados a los } \\
\text { contextos culturales y a las herramientas simbólicas. }\end{array}$
\end{tabular}

\begin{tabular}{ccccc}
\hline IC & Emoción & $\begin{array}{c}\text { Actuación } \\
\text { Docente }\end{array}$ & EG & EE \\
\hline
\end{tabular}

El profesor explica con términos

"técnicos" y

“abstractos” y los

estudiantes se han

desmotivado. No

comprenden

Frustración

Explica "más

didácticamente", sin pedirles

demasiado

$\begin{array}{ll}\text { Estrategia } & \text { Simplificar- } \\ \text { de protección } & \text { facilitar }\end{array}$

Sugiere que use un lenguaje literario y modifica el

podía expresar

lenguaje académico el

significado de un

concepto propio de la

cultura Quechua

Frustración

planteamiento del

trabajo sucesivas

veces

Estrategia

potencialmente Recurso cultura

ajustada

Prueba otras

estrategias más

vivenciales
Estrategia
potencialmente Diversifica ajustada
métodos teórico y no logra
Disgusto
Frustración resultados

En relación a las emociones que los profesores asociaron a los IC dentro de este eje, destacan principalmente las de frustración, disgusto y ansiedad. Percibieron que sus esfuerzos para dar respuesta al problema daban escaso resultado y apuntaron únicamente a resolver la situación inmediata o a evitar el problema.

Como puede observarse (Tabla III), las EG más utilizadas fueron la (C) y (B). En algún caso (2) también identificamos la (D), que permitiría cuestionar la propia actuación, y escapar del esquema habitual de acción y pensamiento. Las EE más utilizadas fueron, en este orden, la simplificación (8), el uso de recursos de la cultura de los estudiantes (7) y la diversificación de métodos (4).

\section{Eje lenguas, expresión y comunicación}

Las emociones asociadas a los incidentes críticos de este eje, fueron principalmente de ansiedad y frustración. Algunos profesores relataron también que experimentaron disgusto y culpa. La frustración y la ansiedad fueron ocasionadas por el escaso manejo de la lengua de algunos estudiantes, lo que se manifestó como un obstáculo para el logro de las metas educativas. En cuanto a la ansiedad, en general, se identificó como fruto de las dificultades para encontrar soluciones a los problemas.

En la mayoría de los casos, los profesores desplegaron una EG de tipo (B), que apuntó a restablecer el equilibrio sin necesidad de tomar consciencia del origen del problema. Los profesores percibieron la situación como irresoluble y pretendían salir rápidamente del 
TABLA IV

Eje lenguas, expresión y comunicación

Definición del eje de conflicto
Conflictos generados por el uso de la lengua vehicular en el contexto universitario, que entra en conflicto con el proceso de apropiación de dicha herramienta como instrumento de pensamiento en relación a la demanda de la actividad universitaria.

\begin{tabular}{lllll}
\hline \multicolumn{1}{c}{ IC } & Emoción & $\begin{array}{l}\text { Actuación } \\
\text { Docente }\end{array}$ & EG & EE \\
\hline $\begin{array}{l}\text { Un grupo de alumnos } \\
\text { expuso un tema sin } \\
\text { entender lo que } \\
\text { exponía }\end{array}$ & $\begin{array}{l}\text { Angustia } \\
\text { Ansiedad }\end{array}$ & $\begin{array}{l}\text { Dejó pasar la } \\
\text { situación y evitó } \\
\text { el problema }\end{array}$ & $\begin{array}{l}\text { Estrategia } \\
\text { localizada }\end{array}$ & No actuar \\
$\begin{array}{l}\text { Los estudiantes no } \\
\text { comprendieron un } \\
\text { texto por su escaso } \\
\text { dominio del lenguaje } \\
\text { utilizado }\end{array}$ & Frustración & $\begin{array}{l}\text { Aunque cree que } \\
\text { el texto era fácil lo } \\
\text { sustituyó por } \\
\text { actividad práctica }\end{array}$ & $\begin{array}{l}\text { Estrategia } \\
\text { localizada }\end{array}$ & $\begin{array}{l}\text { Actividad } \\
\text { práctica }\end{array}$ \\
$\begin{array}{l}\text { Un alumno muestra } \\
\text { muchas dificultades } \\
\text { para expresar un } \\
\text { contenido de la } \\
\text { asignatura debido a su } \\
\text { manera de hablar y } \\
\text { comprender }\end{array}$ & Ansiedad & $\begin{array}{l}\text { Pide a un alumno } \\
\text { mediador que lo } \\
\text { explique en su }\end{array}$ & $\begin{array}{l}\text { Estrategia } \\
\text { idioma } \\
\text { ajustada }\end{array}$ & Recurso cultura \\
\hline
\end{tabular}

paso. Aunque encontramos variedad de EE, fue predominante la de simplificación/facilitación (8). Las decisiones relatadas por los profesores para resolver los IC analizados, tuvieron por finalidad regular la emoción y salir de la situación. Para ello, las acciones predominantes fueron las de evasión o supresión del problema inmediato utilizando estrategias conocidas de simplificación, como volver a un lenguaje más claro, o utilizar palabras más comunes aunque esto signifique abandonar importantes metas educativas.

\section{Eje concepciones del tiempo}

Las emociones asociadas a los IC de este eje fueron disgusto y rabia. En los dos IC de este eje, las EG de resolución estuvieron orientadas a favorecer los objetivos de aprendizaje, lo que propició situaciones de reflexión sobre las situaciones que interpelaban a los profesores abriendo mayores posibilidades de cambio. Las EE más frecuentes fueron la reflexión/mediación (1) y recurso cultura (7) (ver Tabla V).

\section{Eje contextualización}

Frente a los IC ubicados en este eje, los profesores experimentaron principalmente emociones de frustración y disgusto. También mencionaron la culpa por no poder ajustarse mejor a las características del contexto. La EG en estos IC fue de protección y la EE de no actuación.

En la mayoría de IC analizados, no se registraron actuaciones -bien por falta de recursos o por propia decisión-a pesar de que los profesores reconocieron sentirse interpelados.

\section{Eje discriminación}

Frente a los IC registrados en este eje, los profesores experimentaron con más frecuencia rabia, disgusto y miedo, en lugar de la ansiedad y frustración que observábamos en los dos 
TABLA V

Eje concepciones del tiempo

Definición del eje de conflicto
Trata de un conflicto entre sistemas culturales. La universidad tiene una matriz cultural organizada en el tiempo occidental. Entra en conflicto con las concepciones no occidentales.

\begin{tabular}{|c|c|c|c|c|}
\hline IC & Emoción & $\begin{array}{l}\text { Actuación } \\
\text { Docente }\end{array}$ & EG & $\mathbf{E E}$ \\
\hline $\begin{array}{l}\text { Un estudiante llega } \\
\text { una hora tarde al } \\
\text { examen y lo justifica } \\
\text { diciendo que en el } \\
\text { anterior llegó en hora }\end{array}$ & $\begin{array}{l}\text { Disgusto } \\
\text { Rabia }\end{array}$ & $\begin{array}{l}\text { Usa esta situación } \\
\text { para ilustrar las } \\
\text { diferencias } \\
\text { culturales en la } \\
\text { concepción y } \\
\text { reajusta el } \\
\text { programa }\end{array}$ & $\begin{array}{l}\text { Estrategia } \\
\text { potencialmente } \\
\text { ajustada }\end{array}$ & Recurso cultura \\
\hline $\begin{array}{l}\text { No entregan en fecha } \\
\text { los trabajos por que } \\
\text { "todavía" los están } \\
\text { haciendo }\end{array}$ & $\begin{array}{l}\text { Disgusto } \\
\text { Rabia }\end{array}$ & $\begin{array}{l}\text { Reflexiona con los } \\
\text { alumnos } \\
\text { evidenciando el } \\
\text { problema y } \\
\text { llegando a } \\
\text { acuerdos y } \\
\text { reorganiza la } \\
\text { materia }\end{array}$ & $\begin{array}{l}\text { Estrategia } \\
\text { potencialmente } \\
\text { ajustada }\end{array}$ & $\begin{array}{l}\text { Reflexión } \\
\text { Mediación }\end{array}$ \\
\hline
\end{tabular}

TABLA VI

Eje Contextualización

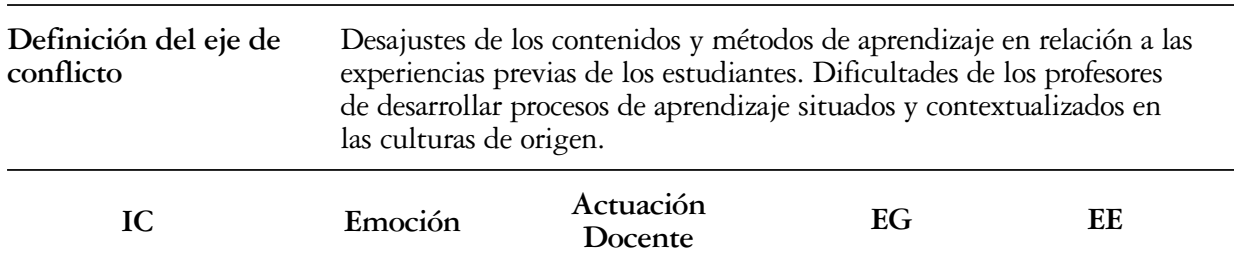

Siente que lo que les está explicando no tiene nada que ver con la realidad de los alumnos

Da una clase teórica y sus alumnos no ven la utilidad de esos conceptos
Trata de pedirles ejemplos de su realidad. No encuentra estrategias y continúa con lo que estaba haciendo

No sabe cómo enfrentarse. $\quad$ Estrategia Diversifica Prueba otras localizada métodos

Estrategia de protección No dispone metodologías

primeros ejes. Aparecieron también sentimientos de culpa y de tristeza frente a situaciones valoradas como injustas.

En varios de los IC analizados las EG que relataron los profesores fueron de tipo (B) evitación o protección, pero las EE fueron más diversas. Dado que los IC tuvieron que ver con relaciones personales, lo que se tradujo muchas veces en un cuestionamiento directo al profesor, éste se escudó en el rol docente y tomó decisiones que lo mantuvieran en el control de la situación. 
572 Cultura y Educación, 2013, 25 (4), pp. 561-576

TABLA VII

Eje discriminación

Definición del eje de conflicto

Situaciones en las que la diferencia se convierte en discriminación.

Intolerancia, prejuicios y actitudes racistas o de confrontación entre grupos.

\begin{tabular}{lllll}
\hline \multicolumn{1}{c}{ IC } & Emoción & $\begin{array}{l}\text { Actuación } \\
\text { Docente }\end{array}$ & EG & EE \\
\hline $\begin{array}{l}\text { El profesor siente que } \\
\text { no hubo empatía con } \\
\text { los estudiantes y sus } \\
\text { estrategias no le } \\
\text { funcionan }\end{array}$ & $\begin{array}{l}\text { Miedo } \\
\text { Frustración }\end{array}$ & $\begin{array}{l}\text { No sabe cómo } \\
\text { enfrentarse. Los } \\
\text { divide en grupos } \\
\text { para poder } \\
\text { manejar la } \\
\text { situación }\end{array}$ & $\begin{array}{l}\text { Estrategia de } \\
\text { protección }\end{array}$ & $\begin{array}{l}\text { Organización del } \\
\text { grupo }\end{array}$ \\
$\begin{array}{l}\text { Los alumnos rechazan } \\
\text { al profesor por ser de } \\
\text { origen indígena }\end{array}$ & $\begin{array}{l}\text { Vergüenza } \\
\text { Rabia }\end{array}$ & No hace nada & $\begin{array}{l}\text { Estrategia de } \\
\text { protección }\end{array}$ & No actuar \\
$\begin{array}{l}\text { No aceptan a una } \\
\text { alumna de rasgos } \\
\text { indígenas en un grupo }\end{array}$ & $\begin{array}{l}\text { Rabia } \\
\text { Disgusto }\end{array}$ & $\begin{array}{l}\text { El profesor se } \\
\text { impone y la } \\
\text { nombra jefa de } \\
\text { grupo }\end{array}$ & $\begin{array}{l}\text { Estrategia } \\
\text { localizada }\end{array}$ & $\begin{array}{l}\text { Organización del } \\
\text { grupo }\end{array}$ \\
$\begin{array}{l}\text { Unos alumnos no } \\
\text { quieren hablar en su } \\
\text { idioma por vergüenza }\end{array}$ & $\begin{array}{l}\text { Frustración } \\
\text { Tristeza }\end{array}$ & $\begin{array}{l}\text { El profesor llama } \\
\text { a la reflexión e } \\
\text { intenta animarlos }\end{array}$ & $\begin{array}{l}\text { Estrategia } \\
\text { localizada }\end{array}$ & $\begin{array}{l}\text { Reflexión- } \\
\text { mediación }\end{array}$ \\
\hline
\end{tabular}

La EE que más mencionaron los profesores en estos IC fue la de reflexión/mediación (1) que corresponde a una respuesta que permite solucionar el IC de forma puntual, pero que no necesariamente evita que vuelva a ocurrir en el futuro.

\section{Eje participación}

Las emociones que relataron los docentes frente los IC de este eje son, una vez más, de frustración y ansiedad, aunque también las hubo de disgusto.

En este eje, la mayor parte de profesores consideró el tema de la participación como un problema de los estudiantes y no como algo inherente a la organización de la actividad de aula.

Como también vimos en otros ejes, las EG que más relataron los profesores fueron de tipo (B) y la EE más frecuente fue la no actuación (9) dejando que fueran los propios estudiantes quienes resolvían el problema.

Cuando relataron alguna actuación, las estrategias fueron EG de tipo (C), es decir, dirigidas a la resolución de la situación concreta pero sin llegar a cuestionar sus actuaciones. Las EE que registramos en este eje fueron la incentivación (5) y la EE simplificación/facilitación (8). Sin embargo no hubo cambios en el esquema de acción.

En síntesis, como hemos observado los profesores relataron la presencia de emociones negativas en todos los IC, siendo la más frecuente el disgusto o rabia (en 15 de los IC), la frustración (12) y la ansiedad y la angustia (10) seguidas de la culpa (6), la tristeza (2) y la impotencia y la vergüenza (1).

\section{DISCUSIÓN Y CONCLUSIONES}

En esta investigación, hemos puesto a prueba un esquema de análisis que nos ha permitido identificar las emociones asociadas por los profesores a los IC referidos a la diversi- 
TABLA VIII

Eje participación

Definición del eje de conflicto
Trata de situaciones de exclusión, la presencia en el contexto universitario no garantiza una igualdad de condiciones de participación y de oportunidades educativas.

\begin{tabular}{lllll}
\hline \multicolumn{1}{c}{ IC } & Emoción & $\begin{array}{c}\text { Actuación } \\
\text { Docente }\end{array}$ & EG & EE \\
\hline $\begin{array}{l}\text { Pide comentarios } \\
\text { sobre una lectura. } \\
\text { Nadie responde }\end{array}$ & $\begin{array}{l}\text { Miedo } \\
\text { Ansiedad }\end{array}$ & $\begin{array}{l}\text { No hace nada, } \\
\text { continúa con la } \\
\text { exposición }\end{array}$ & $\begin{array}{l}\text { Estrategia de } \\
\text { protección }\end{array}$ & No actuar \\
$\begin{array}{l}\text { Surgen situaciones de } \\
\text { silencio y pese a las } \\
\text { preguntas no }\end{array}$ & $\begin{array}{l}\text { Frustración } \\
\text { contestan }\end{array}$ & $\begin{array}{l}\text { Les pide ejemplos } \\
\text { de su vivencia, } \\
\text { sin éxito, desiste }\end{array}$ & $\begin{array}{l}\text { Protección o } \\
\text { evitación y } \\
\text { estrategia } \\
\text { localizada }\end{array}$ & Incentivar \\
$\begin{array}{l}\text { Les toca exponer y } \\
\text { tienen dificultades } \\
\text { para enfrentar la } \\
\text { situación }\end{array}$ & $\begin{array}{l}\text { Ansiedad } \\
\text { Disgusto }\end{array}$ & $\begin{array}{l}\text { Les pide que } \\
\text { expongan en su } \\
\text { propio idioma } \\
\text { pero no se } \\
\text { asegura que los } \\
\text { demás } \\
\text { comprendan }\end{array}$ & Estrategia & Recurso cultura \\
& & localizada & \\
\hline
\end{tabular}

dad sociocultural de sus estudiantes y las estrategias empleadas para su resolución, además de analizar las relaciones entre estas emociones y estrategias y los cambios en la identidad docente.

En cuanto a la identificación de las estrategias, las más frecuentes fueron las Estrategias Generales de tipo (B), y las Estrategias Especificas de reflexión/meditación (1), organización del grupo (6), y no actuación (9).

Los profesores percibieron la diversidad como un problema y esto probablemente incidió en el uso mayoritario de estrategias localizadas (C) unidas a actuaciones de evasión del problema o de autoprotección (B), disminuyendo su eficiencia para resolver los IC.

Las estrategias que más frecuentemente reseñaron sólo permitían gestionar la situación inmediata, pero no resolver el problema de fondo, que percibieron como irresoluble con origen en las condiciones personales de los estudiantes o en elementos externos. Por ello, la combinación de EG y EE resultó poco ajustada a las demandas del contexto dado que aleja al profesor de sus propósitos iniciales permitiéndole sólo modificaciones parciales, que en realidad no cambian la situación.

Las emociones reseñadas, todas ellas negativas, dificultan la acción, distraen de las metas educativas, obstaculizan los procesos de cambio, incrementan las resistencias, afectan en su capacidad de resolución de problemas y disminuyen la toma de riesgos (Kelchtermans, 2005; Lasky, 2005; Reio, 2005; Sutton y Wheatley, 2003). Consecuentemente, en la mayoría de los IC analizados los profesores no quisieron tomar riesgos y se sintieron limitados e inseguros respecto a la diversidad sociocultural.

En relación a la actuación docente, tal como se afirma en trabajos anteriores (Burgum y Bridge, 1997; Monereo, 2010; Monereo et al., 2009), la necesidad de gestionar las emociones fue el detonante de los IC, estuvo en la base de las decisiones a tomar e interpeló alguna dimensión de la identidad de los profesores. Como consecuencia de ello, la opción por una Estrategia General (EG) determinada, respondería a la pregunta ¿Cómo salgo de este problema? Esta primera decisión es determinante, ya que de ella dependen las Estrategias específicas. 
Como comentamos, partimos del supuesto de que las emociones producidas por un IC generan una situación de desequilibrio que, unida a la revisión de la situación, abre la posibilidad de deconstruir y reconstruir la identidad. Sin embargo, si en este momento no hay una actuación intencional que permita retomar el IC esta revisión no se lleva a cabo, se suprime la emoción y la posibilidad de cambio se pierde. Esto es lo que se desprende de los datos analizados. Al no haberse producido un proceso de reflexión sistemático por parte de los profesores respecto a los IC relatados, las EG empleadas fueron inmediatas e hicieron mella en aspectos muy puntuales, lo que derivó en actuaciones reactivas frente a los IC y la elección de estrategias poco eficaces.

Además, pese a la poca efectividad de sus estrategias, en la mayoría de ocasiones, al redescribir la experiencia, los profesores no modificaron sus posiciones iniciales y cuando se produjo algún cambio, éste fue debido a una modificación natural de la situación inicial detectada y no un resultado de una decisión intencional. Fueron excepcionales los IC en los que el despliegue de estrategias y la reflexión posterior permitieron esbozar cambios estructurales en alguna dimensión de la identidad del profesor, y consecuentemente, modificando la manera de enfrentar dichos IC, cambiando su interpretación, emociones y acciones.

En estos casos en los que se abrieron posibilidades de cambio, la identidad del profesor se vio interpelada y se generó un proceso de reflexión, de aprendizaje y de comprensión más profunda de los IC implicados.

Aunque no hemos realizado un análisis transversal para establecer la relación específica entre las percepciones del profesor sobre la diversidad sociocultural y la resolución de los IC, de los datos presentados parece desprenderse la hipótesis de que aquellos profesores que tuvieron más presente el problema de la diversidad cultural, tenían un conocimiento más profundo del tema y habían desarrollado un proceso de reflexión al respecto, eligieron con más frecuencia EG potencialmente ajustadas, aspecto que debería confirmarse en investigaciones futuras.

En cuanto a las limitaciones del estudio, no hemos podido establecer una relación concluyente entre el tipo de IC y las estrategias empleadas dado que ninguna EG o EE fue exclusiva de alguno de los IC; sin embargo, esto nos conduce a señalar la necesidad de que los procesos de formación del profesorado sean situados, ya que no es relevante el tipo de estrategias útiles comúnmente empleadas para contextos de alta diversidad cultural en general, sino que es imprescindible un análisis de casos en relación a una manifestación concreta de un eje de conflicto, que en ese momento debe ser afrontada por un profesor con características identitarias particulares, en un contexto institucional y social específico. Es necesario profundizar en otros estudios, acerca de cómo la identidad influye en la elección estratégica de determinadas prácticas docentes.

\section{Notas}

\footnotetext{
${ }^{1}$ Ría utiliza los términos sensation, sentiments y emotion-types, mientras que Van Veen, utiliza los términos mood, emotions (equivalente a los sentimientos definidos por Ria) y Sentiments. Nosotros utilizaremos Sensación, Emoción básica (para distinguirla de emociones en general que engloba todos los estados emocionales) y Sentimientos (Perinat, 1998; Soldevila, Filella, Ribes y Agulló, 2007).

${ }^{2}$ El primer número que aparece hace referencia al documento primario (17), es decir corresponde a la transcripción de cada profesor entrevistado, el siguiente número es el número de cita en ese documento (17) y el último es el párrafo en el que se ubica la cita (36).
}

\section{Referencias}

Beth R. \& Sharma P. (2008). Struggling toward a transformative model of instruction: It's not so easy! Teaching and Teacher Education, 24, 1070-1082.

Burgum, M. \& BRIDGE, C. (1997). Using critical incidents in professional education to develop skills of reflection and critical thinking. En R. Pospisil \& L. Willcoxson (Eds.), Learning Through Teaching 
(pp. 58-61). Proceedings of the 6th Annual Teaching Learning Forum, Murdoch University, February 1997. Perth: Murdoch University. Consultado 25 de abril de 2010 en: http://sn.curtin.edu.au/tlf/ tlf1997/burgum.html

Cope, P. \& STEPHen, C. (2001). A role for practicing teachers in initial teacher education. Teaching and Teacher Education, 17 (8), 913-924.

Corbin, J. \& Strauss, A. (1990). Grounded theory research: Procedures, canons, and evaluative criteria. Qualitative Sociology, 13 (1), 3-21.

Day, C., Elliot, B. \& Kington, A. (2005). Reform Standards and teacher identity: Challenges of sustaining commitment. Teaching and teachers education, 2, 563-577,

Day, C., Kington, A., Stobart, G. \& Sammons, P. (2006). The personal and professional selves of teachers: stable and unstable identities. British Educational Research Journal, 32 (4), 572-601.

EBbut, D., Robson, R. \& Worral, L, N. (2000). Educational research partnership: differences and tensions at the interface between the professional cultures of practitioners in schools and researches in higher education. Teacher Development, 4, 319-232.

Enyedy, N., Goldberg, J. \& Welsh, K. M. (2006), Complex dilemmas of identity and practice. Science Education, 90 (1), 68-93.

GeE, P. J. (2001). Identity as an Analytic Lens for Research in Education. Review of Research in Education, $25,99-125$.

Heargreaves, A. (1998). The emotional practice of teaching. Teacher and Teaching Education, 14 (8), 835854.

Hopper, B. (2008). Stories We Teach By: Intersections Among Faculty Biography, Student Formation, and Instructional Processes. The American Journal of Occupational Therapy, 62 (2), 228.

Kelchtermans, G. (2005). Teacher's emotions in educational reforms: Self-understanding, vulnerable commitment and micropolitical literacy. Teaching and Teacher Education, 21, 995-1006.

LASKY, S. (2005). A sociocultural approach to understanding teacher identity, agency and professional vulnerability in a context of secondary school reform. Teaching and Teacher Education, 21, 899-916.

Mewborn, D. S. \& Stanulis, R. N. (2000). Making the tacit explicit: teacher educators' values and practices in a co-reform teacher education program. Teacher Education Quarterly, 27 (3), 5-22.

Miles, M. B. \& Huberman, A. M. (1984). Qualitative data analysis, A sourcebook of new methods. Berverly Hills, CA: SAGE.

Monereo, C. (2010). La formación del profesorado: Una pauta para el análisis e intervención a través de incidentes críticos. Revista Iberoamericana de Educación, 52, 149-162.

Monereo, C., Badía, A., Bilbao, G., Cerrato, M. \& Weise, C. (2009). Ser un docente estratégico: cuando cambiar la estrategia no basta. Cultura y educación, 21 (3), 1-20.

Nixon, J. (1996). Professional Identity and the Restructuring of Higer Education. Studies in Higer Education, 21 (1), 5-16.

PANDit, N. R. (1996). The creation of theory: a recent application of the grounded theory method. The Qualitative Report, 2 (4). Consultado 25 de abril de 2010 en: http://www.nova.edu/ssss/QR/QR24/pandit.html

Perinat, A. (1998). Psicología del desarrollo. Un enfoque sistémico. Barcelona: Ediuoc.

Reio, T. G. (2005). Emotions as a lens to explore teacher identity and change: A commentary. Teaching and Teacher Education, 21 (8), 985-993.

Ria, L., Sève, C., Saury, J., Theureu, J. \& Durand, M. (2003). Beginnig Teachers' Situated Emotion: a study of first classroom experiences. Journal of Education for Teaching, 29 (3), 219-233.

Rice, E. H. (2002). The collaboration process in professional development schools results of a metaethnography, 1980-1998". Journal of Teacher Education, 53 (1), 55-67.

Roberts, A. L. (2007). Relaciones entre identidad y aprendizaje. Cultura y Educación, 19 (4), 379-394.

ScotT, C. \& SutTon, R. E. (2009). Emotions and Change During Professional Development for Teachers A Mixed Methods Study. Journal of Mixed Methods Research, 3 (2), 151-171.

Settlage, J., Southerland, S. A., Smith, L. K. \& Ceglie, R. (2009). Constructing a Doubt-Free Teaching Self: Self-Efficacy, Teacher Identity, and Science Instruction Within Diverse Settings. Journal of research in science teaching, 46, 102-125.

SHAPIRO, S. (2010). Revisting the teachers' lounge: Reflections on emotional experience and teacher identity. Teaching and Teacher Education, 26 (3), 616-621.

Soldevila, A., Filella, G., Ribes, R. \& Agulló, M. J. (2007). Una propuesta de contenidos para desarrollar la conciencia y la regulación emocional en la Educación Primaria. Cultura y Educación, 19 (1), 47-59

Sutherland, L., Howard, S. \& Markauskaite, L. (2010). Professional identity creation: Examining the development of beginning preservice teachers' understanding of their work as teachers. Teaching and Teacher Education, 26 (3), 455-465.

Sutton, R. S. \& WheAtley, K. F. (2003). Teachers' emotions and teaching: a review of the literature and directions for future research. Educational Psychology Review, 15 (4), 327-358.

TripP, D. (1994). Teachers' lives, critical incidents and professional practice. Qualitative Studies in Education, 7 (1), 65-76. 
576 Cultura y Educación, 2013, 25 (4), pp. 561-576

Van Veen, K. \& Lasky, S. (2005). Emotions as a lens to explore teacher identity and change: Different theoretical approaches. Teaching and Teacher Education, 21 (8), 895.

Veiravé, D., OJeDa, M., Nuñez, C. \& Delgado, P. (2006). La construcción de la Identidad de los profesores de Enseñanza Media. Biografías de Profesores. Revista Iberoamericana de Educación, 40 (3), 2-11.

WeISE, C. (2011). La atención a contextos de alta diversidad sociocultural en la universidad. Un análisis de la identidad y la práctica docente a través de incidentes críticos. Tesis doctoral inédita. Universidad Autónoma de Barcelona. Barcelona. 